\title{
An Efficient Algorithm for Simulating the Drawdown Stopping Time and the Running Maximum of a Brownian Motion
}

\author{
Angelos Dassios ${ }^{1} \cdot$ Jia Wei Lim² (D)
}

Received: 16 May 2016

Accepted: 3 January 2017 / Published online: 25 January 2017

(c) The Author(s) 2017. This article is published with open access at Springerlink.com

\begin{abstract}
We define the drawdown stopping time of a Brownian motion as the first time its drawdown reaches a duration of length 1 . In this paper, we propose an efficient algorithm to efficiently simulate the drawdown stopping time and the associated maximum at this time. The method is straightforward and fast to implement, and avoids simulating sample paths thus eliminating discretisation bias. We show how the simulation algorithm is useful for pricing more complicated derivatives such as multiple drawdown options.
\end{abstract}

Keywords Drawdown stopping time $\cdot$ Monte Carlo simulation $\cdot$ Multiple drawdown options

Mathematics Subject Classification (2010) $65 \mathrm{C} 05 \cdot 65 \mathrm{C} 50$

\section{Introduction}

Drawdown measures the fall in value of a process from its running maxima, and is frequently used as performance indicators in the fund management industry. There are two dimensions to drawdown risk - the magnitude and duration of the drawdown. Large market drawdowns lead to portfolio losses and liquidity shocks, while a prolonged drawdown period can also lead to high sustained losses. The magnitude of drawdowns has been studied

Jia Wei Lim

j114400@bristol.ac.uk

Angelos Dassios

a.dassios@1se.ac.uk

1 Department of Statistics, London School of Economics, Houghton Street, London, WC2A 2AE, UK

2 School of Mathematics, University of Bristol, Senate House, Tyndall Avenue, Bristol, BS8 1TH, UK 
extensively in the literature. In particular the probabilistic properties of the first time the drawdown amount for a Brownian motion exceeds a certain threshold $a>0$ has been studied in Taylor (1975) and Douady et al. (2000). Insurance contracts designed to insure against the risk of large drawdowns have been introduced in Carr et al. (2011), who proposed a way to hedge the liability. Zhang and Hadjiliadis (2012) studied this stopping time together with the last visit time of the maximum before the drawdown.

Besides the magnitude, the duration of drawdowns is also important as a measure of risk. Recent studies have been done on the duration and frequency of drawdowns. Landriault et al. (2015) derived the joint distribution of the $n^{\text {th }}$ drawdown time, the running maximum, and the value process of the Brownian motion. The duration of drawdowns for Levy models has also been studied by the same authors in Landriault et al. (2017). Dassios and Lim (2016) introduced the drawdown stopping time, which is the first time that the drawdown period exceeds a certain length $D>0$, and obtained the joint Laplace transform of this stopping time and the running maximum of the Brownian motion at this time.

This stopping time is related to the Parisian stopping time, which is the first time the length of the excursions around 0 exceed a certain threshold $D$. The joint Laplace transform of the Parisian stopping time and the value of the Brownian motion is derived in Dassios and $\mathrm{Wu}(2010)$ and the density of the Parisian stopping time is obtained in Dassios and Lim (2013, 2015). The distribution of the Parisian stopping time is key to the pricing of Parisian options, which was introduced by Chesney et al. (1997). Anderluh (2008) proposed a method for simulating the Parisian stopping time using an approximation of the hitting time, and Bernard and Boyle (2011) developed a Monte Carlo method to price discrete Parisian options.

In this paper, we propose an efficient algorithm to simulate jointly the drawdown stopping time and the running maximum at the time. This is done by first simulating a geometric random variable, which determines the number of time intervals less than unit length it takes before the drawdown duration reaches length 1 . The drawdown length and maximum achieved for each of these intervals are then added together. Our method is computationally efficient and straightforward to implement, and avoids simulating sample paths, thus eliminating discretisation bias.

Based on our result, we propose a Monte Carlo method for pricing drawdown options, which are options which pay off an amount proportional to the maximum of the underlying asset when the drawdown duration reaches a prespecified length $D$. This type of options were introduced by Dassios and Lim (2016), who obtained a recursive formula for the joint density of the stopping time and the maximum, and used this to price the options. This new simulation method is faster and computationally more efficient. Like other Monte Carlo methods, the greatest advantage comes when multiple variables are involved. To show its effectiveness, we introduce multiple drawdown options, which pay off an amount proportional to the running maximum at each drawdown time. As the prices of these options involve multiple integrals, our algorithm eliminates the need to evaluate these integrals and provides a fast method to compute the prices of these options.

This paper is organised as follows. Section 2 begins with the mathematical set-up of the problem and introduces a result which lead to the algorithm. Section 3 provides the simulation algorithm and its proof. To verify the accuracy of our algorithm, we compare the numerical results with theoretical properties of the two random variables in Section 4. Section 5 presents the method for pricing drawdown options and multiple drawdown options using Monte Carlo simulation. 


\section{Joint Laplace Transform of $\tau$ and $M_{\tau}$}

Let $\left(\Omega, \mathcal{F},\left(\mathcal{F}_{t}\right)_{t \geq 0}, \mathbb{P}\right)$ be a filtered probability space and let $W$ be a standard Brownian motion adapted to $\left(\mathcal{F}_{t}\right)_{t \geq 0}$ with $W_{0}=0$. Its maximum process is

$$
M_{t}=\max _{s \leq t} W_{s}
$$

and its drawdown process is

$$
Y_{t}=M_{t}-W_{t}
$$

We denote by $U_{t}$ the time elapsed since the last time the maximum is achieved,

$$
U_{t}=t-\sup \left\{s \leq t \mid M_{t}=W_{s}\right\}=t-\sup \left\{s \leq t \mid Y_{t}=0\right\} .
$$

Define the stopping time

$$
\tau_{D}=\inf \left\{t \geq 0 \mid U_{t}=D\right\} .
$$

This is the first time the duration of drawdowns exceeds a certain threshold $D>0$. Without loss of generality, we set $D=1$ and drop its notation.

Theorem 2.1 The joint Laplace transform of $\tau$ and $M_{\tau}$ can be written as

$$
E\left(e^{-\beta \tau} e^{-\gamma M_{\tau}}\right)=\frac{\frac{2}{\pi} e^{-\beta} \int_{0}^{\infty} \int_{0}^{1} e^{-\gamma m} e^{-\beta s} \frac{m}{2 s^{3 / 2}} e^{-\frac{m^{2}}{2 s}} d s d m}{1-\frac{\pi-2}{\pi} \int_{0}^{\infty} e^{-\gamma m} e^{-\beta} \int_{0}^{1} e^{-\beta t} f(t, m) d t d m}
$$

Furthermore, we have

$$
f(t, m)=g(t) w(m \mid t),
$$

where $g(t)$ is a proper density function over $(0,1)$ and $w(m \mid t)$ is a conditional density over $(0, \infty)$ given by

$$
g(t)=\frac{1-t}{(\pi-2)(t+1) \sqrt{t}},
$$

and

$$
\begin{aligned}
w(m \mid t)= & \frac{\sqrt{2 \pi} \sqrt{t}}{(1-t) \sqrt{t+1}} e^{-\frac{m^{2}}{2(t+1)}}\left(1-\frac{m^{2}}{t+1}\right)\left(\mathcal{N}\left(\frac{m}{\sqrt{t(t+1)}}\right)-\mathcal{N}\left(m \sqrt{\frac{t}{t+1}}\right)\right) \\
& +\frac{m}{1-t^{2}}\left(e^{-\frac{m^{2}}{2}}-t e^{-\frac{m^{2}}{2 t}}\right) .
\end{aligned}
$$

Proof From Dassios and Lim (2016), we have

$$
E\left(e^{-\beta \tau} e^{-\gamma M_{\tau}}\right)=\frac{e^{-\beta}}{\gamma \sqrt{\frac{\pi}{2}}+\beta \int_{0}^{1} e^{-\beta t} \frac{1}{\sqrt{t}} d t+e^{-\beta}} .
$$


Multiply both the numerator and denominator by

$$
\int_{0}^{\infty} \int_{0}^{1} e^{-\gamma m} e^{-\beta s} \frac{m}{s^{3 / 2}} e^{-\frac{m^{2}}{2 s}} d s d m
$$

In the denominator, the first term is

$$
\begin{aligned}
\gamma & \sqrt{\frac{\pi}{2}} \int_{0}^{\infty} \int_{0}^{1} e^{-\gamma m} e^{-\beta s} \frac{m}{s^{3 / 2}} e^{-\frac{m^{2}}{2 s}} d s d m \\
& =\gamma \sqrt{\frac{\pi}{2}} \int_{0}^{\infty}\left(\int_{0}^{\infty} e^{-\gamma m} e^{-\beta s} \frac{m}{s^{3 / 2}} e^{-\frac{m^{2}}{2 s}} d s-\int_{1}^{\infty} e^{-\gamma m} e^{-\beta s} \frac{m}{s^{3 / 2}} e^{-\frac{m^{2}}{2}} d s\right) d m \\
& =\frac{\pi \gamma}{\gamma+\sqrt{2 \beta}}-\sqrt{\frac{\pi}{2}} \int_{0}^{\infty} \int_{1}^{\infty} e^{-\gamma m} e^{-\beta s}\left(\frac{1}{s^{3 / 2}} e^{-\frac{m^{2}}{2 s}}-\frac{m^{2}}{s^{5 / 2}} e^{-\frac{m^{2}}{2 s}}\right) d s d m .
\end{aligned}
$$

The second term in the denominator is

$$
\begin{aligned}
\beta \int_{0}^{\infty} & e^{-\gamma m}\left(\int_{0}^{1} e^{-\beta s} \frac{1}{\sqrt{s}} d s\right)\left(\int_{0}^{1} e^{-\beta u} \frac{m}{u^{3 / 2}} e^{-\frac{m^{2}}{2 u}} d u\right) d m \\
= & \beta \int_{0}^{\infty} e^{-\gamma m}\left(\int_{0}^{1} e^{-\beta t} \int_{0}^{t} \frac{1}{\sqrt{s}} \frac{m}{(t-s)^{3 / 2}} e^{-\frac{m^{2}}{2(t-s)}} d s d t\right. \\
& \left.+\int_{1}^{2} e^{-\beta t} \int_{t-1}^{1} \frac{1}{\sqrt{s}} \frac{m}{(t-s)^{3 / 2}} e^{-\frac{m^{2}}{2(t-s)}} d s d t\right) d m \\
= & \beta \int_{0}^{\infty} e^{-\gamma m} \int_{0}^{1} e^{-\beta t} \sqrt{\frac{2 \pi}{t}} e^{-\frac{m^{2}}{2 t}} d t d m \\
& +\beta \int_{0}^{\infty} e^{-\gamma m} \int_{1}^{2} e^{-\beta t}\left(2 \sqrt{\frac{2 \pi}{t}} e^{-\frac{m^{2}}{2 t}}\left(\mathcal{N}\left(m \sqrt{\frac{1}{t(t-1)}}\right)-\mathcal{N}\left(m \sqrt{\frac{t-1}{t}}\right)\right)\right) d t d m
\end{aligned}
$$

Equation 2.13 is equal to

$$
\begin{aligned}
\beta & \int_{0}^{\infty} e^{-\gamma m} \int_{0}^{1} e^{-\beta t} \sqrt{\frac{2 \pi}{t}} e^{-\frac{m^{2}}{2 t}} d t \\
= & \beta \int_{0}^{\infty} e^{-\gamma m} \int_{0}^{\infty} e^{-\beta t} \sqrt{\frac{2 \pi}{t}} e^{-\frac{m^{2}}{2 t}} d t-\beta \int_{0}^{\infty} e^{-\gamma m} \int_{1}^{\infty} e^{-\beta t} \sqrt{\frac{2 \pi}{t}} e^{-\frac{m^{2}}{2 t}} d t d m \\
= & \frac{\pi \sqrt{2 \beta}}{\gamma+\sqrt{2 \beta}}-\int_{0}^{\infty} e^{-\gamma m} \int_{1}^{\infty} e^{-\beta t}\left(-\sqrt{\frac{\pi}{2 t^{3}}} e^{-\frac{m^{2}}{2 t}}+\sqrt{\frac{\pi}{2 t^{5}}} m^{2} e^{-\frac{m^{2}}{2 t}}\right) d t d m \\
& -\int_{0}^{\infty} e^{-\gamma m} e^{-\beta \sqrt{2 \pi}} e^{-\frac{m^{2}}{2}}
\end{aligned}
$$


and Eq. 2.14 is equal to

$$
\begin{aligned}
\beta \int_{0}^{\infty} & e^{-\gamma m} \int_{1}^{2} e^{-\beta t}\left(2 \sqrt{\frac{2 \pi}{t}} e^{-\frac{m^{2}}{2 t}}\left(\mathcal{N}\left(m \sqrt{\frac{1}{t(t-1)}}\right)-\mathcal{N}\left(m \sqrt{\frac{t-1}{t}}\right)\right)\right) d t d m \\
= & \int_{0}^{\infty} e^{-\gamma m}\left[\frac{\beta e^{-\beta t}}{-\beta}\left(2 \sqrt{\frac{2 \pi}{t}} e^{-\frac{m^{2}}{2}}\left(\mathcal{N}\left(m \sqrt{\frac{1}{t(t-1)}}\right)-\mathcal{N}\left(m \sqrt{\frac{t-1}{t}}\right)\right)\right)\right]_{1}^{2} d m \\
& +\int_{0}^{\infty} e^{-\gamma m} \int_{1}^{2} e^{-\beta t} h(t, m) d t d m \\
= & \int_{0}^{\infty} e^{-\gamma m} e^{-\beta \sqrt{2 \pi} e^{-\frac{m^{2}}{2}}} d m-\int_{0}^{\infty} e^{-\gamma m} \int_{1}^{2} e^{-\beta t} h(t, m) d t d m \\
= & \int_{0}^{\infty} e^{-\gamma m} e^{-\beta \sqrt{2 \pi} e^{-\frac{m^{2}}{2}}} d m-\int_{0}^{\infty} e^{-\gamma m} e^{-\beta} \int_{0}^{1} e^{-\beta t} h(t+1, m) d t d m,
\end{aligned}
$$

where for simplicity we have defined

$$
\begin{aligned}
h(t, m)= & 2 \sqrt{2 \pi}\left(\frac{1}{2 t^{3 / 2}} e^{-\frac{m^{2}}{2 t}}-\frac{1}{\sqrt{t}} e^{-\frac{m^{2}}{2 t}} \frac{m^{2}}{2 t^{2}}\right)\left(\mathcal{N}\left(m \sqrt{\frac{1}{t(t-1)}}\right)-\mathcal{N}\left(m \sqrt{\frac{t-1}{t}}\right)\right) \\
& +\frac{1}{\sqrt{t}} e^{-\frac{m^{2}}{2 t}}\left(\frac{m(2 t-1)}{(t(t-1))^{3 / 2}} e^{-\frac{m^{2}}{2 t(t-1)}}+\frac{m}{t^{3 / 2} \sqrt{t-1}} e^{-\frac{m^{2}(t-1)}{2 t}}\right) .
\end{aligned}
$$

Hence, we have

$$
\begin{aligned}
E\left(e^{-\beta \tau} e^{-\gamma M_{\tau}}\right) & =\frac{e^{-\beta} \int_{0}^{\infty} \int_{0}^{1} e^{-\gamma m} e^{-\beta s} \frac{m}{s^{3 / 2}} e^{-\frac{m^{2}}{2 s}} d s d m}{\pi-\int_{0}^{\infty} e^{-\gamma m} e^{-\beta} \int_{0}^{1} e^{-\beta t}\left(h(t+1, m)-\frac{m}{t^{3 / 2}} e^{-\frac{m^{2}}{2 t}}\right) d t d m} \\
& =\frac{\frac{2}{\pi} e^{-\beta} \int_{0}^{\infty} \int_{0}^{1} e^{-\gamma m} e^{-\beta s} \frac{m}{2 s^{3 / 2}} e^{-\frac{m^{2}}{2 s}} d s d m}{1-\frac{\pi-2}{\pi} \int_{0}^{\infty} e^{-\gamma m} e^{-\beta} \int_{0}^{1} e^{-\beta t} f(t, m) d t d m}
\end{aligned}
$$

where

$$
\begin{aligned}
f(t, m)= & \frac{1}{\pi-2} \frac{\sqrt{2 \pi}}{(t+1)^{3 / 2}} e^{-\frac{m^{2}}{2(t+1)}}\left(1-\frac{m^{2}}{t+1}\right)\left(\mathcal{N}\left(\frac{m}{\sqrt{t(t+1)}}\right)-\mathcal{N}\left(m \sqrt{\frac{t}{t+1}}\right)\right) \\
& +\frac{1}{\pi-2} \frac{m}{\sqrt{t}(t+1)^{2}}\left(e^{-\frac{m^{2}}{2}}-t e^{-\frac{m^{2}}{2 t}}\right) \\
= & g(t) w(m \mid t) .
\end{aligned}
$$


To see that $f(t, m) \geq 0 \forall t>0, m>0$, we use Hermite-Hadamard's Inequality, which states that if a function $h:[a, b] \rightarrow \mathbb{R}$ is convex, then

$$
\frac{1}{b-a} \int_{a}^{b} h(x) d x \leq \frac{h(a)+h(b)}{2} .
$$

Hence we have

$$
\begin{aligned}
\sqrt{2 \pi}\left(\mathcal{N}\left(\frac{m}{\sqrt{t(t+1)}}\right)-\mathcal{N}\left(m \sqrt{\frac{t}{t+1}}\right)\right) & =\int_{m \sqrt{\frac{t}{t+1}}}^{\frac{m}{\sqrt{t(t+1)}}} e^{-\frac{x^{2}}{2}} d x \\
& \leq \frac{m(1-t)}{\sqrt{t(t+1)}} \frac{e^{-\frac{m^{2}}{2 t(t+1)}}+e^{-\frac{m^{2} t}{2(t+1)}}}{2} .
\end{aligned}
$$

Hence,

$$
\begin{aligned}
\frac{\sqrt{2 \pi}}{(t+1)^{3 / 2}} & e^{-\frac{m^{2}}{2(t+1)}}\left(\mathcal{N}\left(\frac{m}{\sqrt{t(t+1)}}\right)-\mathcal{N}\left(m \sqrt{\frac{t}{t+1}}\right)\right) \\
& \leq \frac{m(1-t)}{\sqrt{t}(t+1)^{2}} \frac{e^{-\frac{m^{2}}{2 t}}+e^{-\frac{m^{2}}{2}}}{2} \\
& =\frac{m}{\sqrt{t}(t+1)^{2}} \frac{1}{2}\left(\left(e^{-\frac{m^{2}}{2}}-t e^{-\frac{m^{2}}{2 t}}\right)+\left(e^{-\frac{m^{2}}{2 t}}-t e^{-\frac{m^{2}}{2}}\right)\right) \\
& \leq \frac{m}{\sqrt{t}(t+1)^{2}}\left(e^{-\frac{m^{2}}{2}}-t e^{-\frac{m^{2}}{2 t}}\right),
\end{aligned}
$$

where the last equality follows since $0<t<1$. Now, when $\frac{m^{2}}{t+1} \leq 1$, we have

$$
\begin{gathered}
\frac{m^{2}}{t+1} \frac{\sqrt{2 \pi}}{(t+1)^{3 / 2}} e^{-\frac{m^{2}}{2(t+1)}}\left(\mathcal{N}\left(\frac{m}{\sqrt{t(t+1)}}\right)-\mathcal{N}\left(m \sqrt{\frac{t}{t+1}}\right)\right) \\
\leq \frac{m}{\sqrt{t}(t+1)^{2}}\left(e^{-\frac{m^{2}}{2}}-t e^{-\frac{m^{2}}{2 t}}\right),
\end{gathered}
$$

and thus

$$
\begin{gathered}
\left(1-\frac{m^{2}}{t+1}\right) \frac{\sqrt{2 \pi}}{(t+1)^{3 / 2}} e^{-\frac{m^{2}}{2(t+1)}}\left(\mathcal{N}\left(\frac{m}{\sqrt{t(t+1)}}\right)-\mathcal{N}\left(m \sqrt{\frac{t}{t+1}}\right)\right) \\
\geq \frac{-m}{\sqrt{t}(t+1)^{2}}\left(e^{-\frac{m^{2}}{2}}-t e^{-\frac{m^{2}}{2 t}}\right)
\end{gathered}
$$


and we are done. For $1<\frac{m^{2}}{t+1} \leq 2$, we have $0<\frac{m^{2}}{t+1}-1 \leq 1$ and we have the same result. Finally for $\frac{m^{2}}{t+1}>2$, we have $\frac{m^{2}}{t+1}-1>1$, and hence we have

$$
\begin{aligned}
& \left(\frac{m^{2}}{t+1}-1\right) \frac{\sqrt{2 \pi}}{(t+1)^{3 / 2}} e^{-\frac{m^{2}}{2(t+1)}}\left(\mathcal{N}\left(\frac{m}{\sqrt{t(t+1)}}\right)-\mathcal{N}\left(m \sqrt{\frac{t}{t+1}}\right)\right) \\
& \leq\left(\frac{m^{2}}{t+1}-1\right) \frac{m(1-t)}{\sqrt{t}(t+1)^{2}} \frac{e^{-\frac{m^{2}}{2 t}}+e^{-\frac{m^{2}}{2}}}{2} \\
& =\frac{m}{\sqrt{t}(t+1)^{2}}\left(\frac{m^{2}}{t+1}-\frac{m^{2} t}{t+1}-1+t\right) \frac{e^{-\frac{m^{2}}{2 t}}+e^{-\frac{m^{2}}{2}}}{2} \\
& =\frac{m}{\sqrt{t}(t+1)^{2}} \frac{t^{2}-m^{2} t+m^{2}-1}{1+t} \frac{e^{-\frac{m^{2}}{2 t}}+e^{-\frac{m^{2}}{2}}}{2} \\
& =\frac{m}{\sqrt{t}(t+1)^{2}} \frac{\left(t-\frac{m^{2}}{2}\right)^{2}-\left(\frac{m^{2}}{2}-1\right)^{2}}{1+t} \frac{e^{-\frac{m^{2}}{2 t}}+e^{-\frac{m^{2}}{2}}}{2} \\
& \leq \frac{m}{\sqrt{t}(t+1)^{2}} \frac{1-t^{2}}{1+t} \frac{e^{-\frac{m^{2}}{2 t}}+e^{-\frac{m^{2}}{2}}}{2} \\
& =\frac{m}{\sqrt{t}(t+1)^{2}}(1-t) \frac{e^{-\frac{m^{2}}{2 t}}+e^{-\frac{m^{2}}{2}}}{2} \\
& \leq \frac{m}{\sqrt{t}(t+1)^{2}}\left(e^{-\frac{m^{2}}{2}}-t e^{-\frac{m^{2}}{2 t}}\right),
\end{aligned}
$$

where for the step from Eqs. 2.46 to 2.47 , we observe that $t-\frac{m^{2}}{2}<-1$ and $\frac{m^{2}}{2}-1>t$. Taking the negative of both sides, we see that $f(t, m) \geq 0$.

To see that $\int_{0}^{\infty} w(m \mid t)=1$, we have

$$
\begin{aligned}
w(m \mid t)= & \frac{\sqrt{2 \pi} \sqrt{t}}{(1-t) \sqrt{t+1}} e^{-\frac{m^{2}}{2(t+1)}}\left(1-\frac{m^{2}}{t+1}\right)\left(\mathcal{N}\left(\frac{m}{\sqrt{t(t+1)}}\right)-\mathcal{N}\left(m \sqrt{\frac{t}{t+1}}\right)\right) \\
& +\frac{m}{1-t^{2}}\left(e^{-\frac{m^{2}}{2}}-t e^{-\frac{m^{2}}{2 t}}\right) \\
= & \frac{\sqrt{t}}{(1-t) \sqrt{t+1}}\left(1-\frac{m^{2}}{t+1}\right) \int_{0}^{\infty} e^{-\frac{x^{2}}{2}}\left(e^{-\frac{m^{2}}{2}} e^{-\sqrt{\frac{t}{t+1}} m x}-e^{-\frac{m^{2}}{2 t}} e^{-\frac{1}{\sqrt{t(t+1)}} m x}\right) d x \\
& +\frac{m}{1-t^{2}}\left(e^{-\frac{m^{2}}{2}}-t e^{-\frac{m^{2}}{2 t}}\right) .
\end{aligned}
$$


The integral of Eq. 2.52 over $m$ is 0 since

$$
\begin{aligned}
\int_{0}^{\infty}(1 & \left.-\frac{m^{2}}{t+1}\right) \int_{0}^{\infty} e^{-\frac{x^{2}}{2}}\left(e^{-\frac{m^{2}}{2}} e^{-\sqrt{\frac{t}{t+1}} m x}-e^{-\frac{m^{2}}{2 t}} e^{-\frac{1}{\sqrt{t(t+1)}} m x}\right) d x d m \\
= & \int_{0}^{\infty}\left(1-\frac{m^{2}}{t+1}\right) \int_{0}^{\infty} e^{-\frac{x^{2}}{2}} e^{-\frac{m^{2}}{2}} e^{-\sqrt{\frac{t}{t+1}} m x} d x d m \\
& +\int_{0}^{\infty}\left(1-\frac{m^{2}}{t+1}\right) \int_{0}^{\infty} e^{-\frac{x^{2}}{2}} e^{-\frac{m^{2}}{2 t}} e^{-\sqrt{\frac{1}{t(t+1)}} m x} d x d m \\
= & \int_{0}^{\infty}\left(1-\frac{m^{2}}{t}\right) \int_{0}^{\infty} e^{-\frac{x^{2}}{2}} e^{-\frac{m^{2}(t+1)}{2 t}} e^{-m x} d x d m \\
& +\int_{0}^{\infty}\left(1-m^{2} t\right) \int_{0}^{\infty} e^{-\frac{x^{2}}{2}} e^{-\frac{m^{2}(t+1)}{2}} e^{-m x} d x d m \\
= & \int_{0}^{\infty}\left(1-\frac{m^{2}}{t}\right) \int_{m}^{\infty} e^{-\frac{x^{2}}{2}} e^{-\frac{m^{2}}{2 t}} d x d m+\int_{0}^{\infty}\left(1-m^{2} t\right) \int_{m}^{\infty} e^{-\frac{x^{2}}{2}} e^{-\frac{m^{2} t}{2}} d x d m \\
= & \int_{0}^{\infty} e^{-\frac{x^{2}}{2}} \int_{0}^{x}\left(\left(1-\frac{m^{2}}{t}\right) e^{-\frac{m^{2}}{2 t}}+\left(1-m^{2} t\right) e^{-\frac{m^{2} t}{2}}\right) d m d x \\
= & \int_{0}^{\infty} e^{-\frac{x^{2}}{2}} \int_{0}^{x}\left(e^{-\frac{m^{2}}{2 t}}+e^{-\frac{m^{2} t}{2}}\right) d m d x-\int_{0}^{\infty} e^{-\frac{x^{2}}{2}} \int_{0}^{x}\left(\frac{m^{2}}{t} e^{-\frac{m^{2}}{2 t}}+m^{2} t e^{-\frac{m^{2} t}{2}}\right) d m d x \\
= & 0,
\end{aligned}
$$

where the last step is obtained after integration by parts on the second term. Hence,

$$
\begin{aligned}
\int_{0}^{\infty} w(m \mid t) d m & =\int_{0}^{\infty} \frac{m}{1-t^{2}}\left(e^{-\frac{m^{2}}{2}}-t e^{-\frac{m^{2}}{2 t}}\right) \\
& =1 .
\end{aligned}
$$

Furthermore, it is easy to check that

$$
\int_{0}^{1} g(t) d t=1
$$

Hence, $g(t), w(m \mid t)$ and thus $f(t, m)$ are proper density functions.

\section{Simulation Algorithm}

The representation of the Laplace Transform given in Theorem 2.1 gives us an algorithm for simulating the pair of random variables $\left(\tau, M_{\tau}\right)$. They can be sampled from a Compound Geometric distribution using the following algorithm. The acceptance-rejection schemes used in the algorithm are very efficient, needing only a small expected number of iterations for each sample.

Algorithm 3.1 The simulation algorithm for one sample of $\left(\tau, M_{\tau}\right)$ is given as follow:

1. Generate a Geometric random variable $N$ with

$$
P(N=n)=\frac{2}{\pi}\left(\frac{\pi-2}{\pi}\right)^{n}, \quad n=0,1,2, \ldots
$$


2. Simulate a random variable $Y_{0}$ with density

$$
g_{0}(t)=\frac{1}{2 \sqrt{t}}, \quad 0 \leq t \leq 1
$$

and let $T_{0}=Y_{0}+1$.

3. Let $t=T_{0}$, and simulate a random variable $M_{0}$ with density

$$
w_{0}(m \mid t)=\frac{m}{t} e^{-\frac{m^{2}}{2 t}}, \quad m \geq 0 .
$$

This is a Rayleigh distribution with parameter $\sqrt{t}$, and can be generated using the inverse transform method $M_{0}=\sqrt{-2 t \log (U)}$, where $U \sim U(0,1)$.

4. For $n=N$, simulate the sequence of random variables $Y_{1}, \ldots, Y_{n}$ each with density

$$
g(t)=\frac{1-t}{(\pi-2)(t+1) \sqrt{t}}, \quad 0 \leq t \leq 1,
$$

and take $T_{i}=Y_{i}+1$. This can be implemented using an acceptance-rejection scheme with an envelope density

$$
g_{1}(t)=\frac{1}{\sqrt{t}}-1, \quad 0 \leq t \leq 1 .
$$

5. For each $i=1, \ldots, n$, let $t=Y_{i}$ and generate $M_{i}$ with density

$$
\begin{aligned}
w(m \mid t)= & \frac{\sqrt{2 \pi} \sqrt{t}}{(1-t) \sqrt{t+1}} e^{-\frac{m^{2}}{2(t+1)}}\left(1-\frac{m^{2}}{t+1}\right)\left(\mathcal{N}\left(\frac{m}{\sqrt{t(t+1)}}\right)-\mathcal{N}\left(m \sqrt{\frac{t}{t+1}}\right)\right) \\
& +\frac{m}{1-t^{2}}\left(e^{-\frac{m^{2}}{2}}-t e^{-\frac{m^{2}}{2 t}}\right) .
\end{aligned}
$$

This can be implemented using an acceptance-rejection scheme with the envelope density

$$
\begin{aligned}
w_{1}(m \mid t)= & \frac{m}{c(t) 2(t+3)} \frac{t+3}{t+1} e^{-\frac{m^{2}(t+3)}{2(t+1)}}+\frac{m t}{c(t) 2(3 t+1)} \frac{3 t+1}{t(t+1)} e^{-\frac{m^{2}(3 t+1)}{2 t(t+1)}} \\
& +\frac{1}{c(t)(t+1)} m e^{-\frac{m^{2}}{2}}+\frac{t}{c(t)(t+1)} \frac{m\left(e^{-\frac{m^{2}}{2}}-e^{-\frac{m^{2}}{2 t}}\right)}{1-t}
\end{aligned}
$$

A random variable sampled from the envelope density $w_{1}(m \mid t)$ can be generated as follows:

(a) Let

$$
c(t)=1+\frac{1}{2(t+3)}+\frac{t}{2(3 t+1)},
$$

and define

$$
\begin{aligned}
& q_{1}(t)=\frac{1}{c(t) 2(t+3)} \\
& q_{2}(t)=q_{1}(t)+\frac{t}{c(t) 2(3 t+1)} \\
& q_{3}(t)=q_{2}(t)+\frac{1}{c(t)(t+1)} .
\end{aligned}
$$


(b) Generate three uniform $(0,1)$ random variables $V_{1}, V_{2}$ and $U_{1}$ and take

$$
\begin{aligned}
X= & -2 \log \left(V_{1}\right)\left(\frac{t+1}{t+3} \mathbf{1}_{\left\{U_{1}<q_{1}(t)\right\}}+\frac{t(t+1)}{3 t+1)} \mathbf{1}_{\left\{q_{1}(t)<U_{1}<q_{2}(t)\right\}}+\mathbf{1}_{\left\{q_{2}(t)<U_{1}\right\}}\right) \\
& -2 \log \left(V_{2}\right) t \mathbf{1}_{\left\{q_{3}(t)<U_{1}\right\}} .
\end{aligned}
$$

Then $M_{i}=\sqrt{X}$.

6. The drawdown stopping time $\tau$ is

$$
\tau=T_{0}+T_{1}+T_{2}+\ldots+T_{n},
$$

and the maximum at this time $M_{\tau}$ is

$$
M_{\tau}=M_{0}+M_{1}+\ldots+M_{n},
$$

and for $n=0$ would just be $\tau=T_{0}$ and $M_{\tau}=M_{0}$.

Proof It is clear from Theorem 2.1 that the pair $\left(\tau, M_{\tau}\right)$ has a bivariate compound Geometric distribution with parameter $p=\frac{2}{\pi}$. In particular, we have

$$
E\left(e^{-\beta \tau-\gamma M_{\tau}}\right)=E\left(e^{-\sum_{i=0}^{N}\left(T_{i}+M_{i}\right)}\right)
$$

where $N \sim$ Geometric $(p)$, and the joint density of $\left(T_{0}-1, M_{0}\right)$ is

$$
f_{0,0}(t, m)=\frac{m}{2 t^{3 / 2}} e^{-\frac{m^{2}}{2 t}}=g_{0}(t) w_{0}(m \mid t),
$$

which gives rise to steps 2 and 3 , and the joint density of $\left(T_{i}-1, M_{i}\right)$ is

$$
f(t, m)=g(t) w(m \mid t),
$$

which explains steps 4 and 5 .

The envelope density used in the acceptance-rejection scheme in step 4 can be derived as follows:

$$
\begin{aligned}
g(t) & =\frac{1-t}{(\pi-2)(t+1) \sqrt{t}} \\
& =g_{1}(t) \frac{1+\sqrt{t}}{(\pi-2)(1+t)} \\
& \leq g_{1}(t) \frac{1+\sqrt{t^{*}}}{(\pi-2)\left(1+t^{*}\right)} .
\end{aligned}
$$

where $t^{*}=(\sqrt{2}-1)^{2}$. Hence the expected number of iterations required for each sample of $T_{i}, i=1,2, \ldots, n$ is 1.057 . 
To derive the acceptance-rejection scheme in step 5, we first have the following inequality

$$
\begin{aligned}
& \frac{\sqrt{2 \pi t}}{(1-t) \sqrt{t+1}} e^{-\frac{m^{2}}{2(t+1)}}\left(1-\frac{m^{2}}{t+1}\right)\left(\mathcal{N}\left(\frac{m}{\sqrt{t(t+1)}}\right)-\mathcal{N}\left(m \sqrt{\frac{t}{t+1}}\right)\right) \\
& \leq \frac{\sqrt{2 \pi t}}{(1-t) \sqrt{t+1}} e^{-\frac{m^{2}}{2(t+1)}} e^{-\frac{m^{2}}{t+1}}\left(\mathcal{N}\left(\frac{m}{\sqrt{t(t+1)}}\right)-\mathcal{N}\left(m \sqrt{\frac{t}{t+1}}\right)\right) \\
& \leq \frac{m}{2(t+1)}\left(e^{-\frac{m^{2}}{2}}+e^{-\frac{m^{2}}{2 t}}\right) e^{-\frac{m^{2}}{t+1}} \\
& \quad=\frac{m}{2(t+1)}\left(e^{-\frac{m^{2}(t+3)}{2(t+1)}}+e^{-\frac{m^{2}(3 t+1)}{2 t(t+1)}}\right)
\end{aligned}
$$

where we have used the Hermite-Hadamard Inequality as in Eq. 2.31. Hence, we have

$$
\begin{aligned}
w(m \mid t) \leq & \frac{m}{2(t+1)}\left(e^{-\frac{m^{2}(t+3)}{2(t+1)}}+e^{-\frac{m^{2}(3 t+1)}{2 t(t+1)}}\right)+\frac{m}{1-t^{2}}\left(e^{-\frac{m^{2}}{2}}-t e^{-\frac{m^{2}}{2 t}}\right) \\
= & \frac{m}{2(t+1)}\left(e^{-\frac{m^{2}(t+3)}{2(t+1)}}+e^{-\frac{m^{2}(3 t+1)}{2 t(t+1)}}+2 e^{-\frac{m^{2}}{2}}\right)+\frac{t}{t+1} \frac{m\left(e^{-\frac{m^{2}}{2}}-e^{-\frac{m^{2}}{2 t}}\right)}{1-t} \\
= & \frac{m}{2(t+3)} \frac{t+3}{t+1} e^{-\frac{m^{2}(t+3)}{2(t+1)}}+\frac{m t}{2(3 t+1)} \frac{3 t+1}{t(t+1)} e^{-\frac{m^{2}(3 t+1)}{2 t(t+1)}} \\
& +\frac{1}{t+1} m e^{-\frac{m^{2}}{2}}+\frac{t}{t+1} \frac{m\left(e^{-\frac{m^{2}}{2}}-e^{-\frac{m^{2}}{2 t}}\right)}{1-t} .
\end{aligned}
$$

Letting

$$
c(t)=\frac{1}{2(t+3)}+\frac{t}{2(3 t+1)}+\frac{1}{t+1}+\frac{t}{t+1}=1+\frac{1}{2(t+3)}+\frac{t}{2(3 t+1)},
$$

we have the envelope density

$$
\begin{aligned}
w_{1}(m \mid t)= & \frac{m}{c(t) 2(t+3)} \frac{t+3}{t+1} e^{-\frac{m^{2}(t+3)}{2(t+1)}}+\frac{m t}{c(t) 2(3 t+1)} \frac{3 t+1}{t(t+1)} e^{-\frac{m^{2}(3 t+1)}{2 t(t+1)}} \\
& +\frac{1}{c(t)(t+1)} m e^{-\frac{m^{2}}{2}}+\frac{t}{c(t)(t+1)} \frac{m\left(e^{-\frac{m^{2}}{2}}-e^{-\frac{m^{2}}{2 t}}\right)}{1-t}
\end{aligned}
$$

which can be generated as in step 5 . The average number of iterations needed to generate one sample of $M_{i}$ given $T_{i}=t$ is $c(t)$, and this has expectation

$$
\int_{0}^{1} c(t) \frac{1}{\pi-2} \frac{1-t}{(1+t) \sqrt{t}} d t=1.201
$$

We then sum up the variables to get $\tau$ and $M_{\tau}$ in the last step. 


\section{Numerical Studies}

To verify the accuracy of our algorithm, we compare the sample moments of the simulated $\tau$ and $M_{\tau}$ with the theoretical formulas obtained from the marginal distributions. Firstly, if we set $\beta=0$ in Eq. 2.6, we get

$$
E\left(e^{-\gamma M_{\tau}}\right)=\frac{1}{\gamma \sqrt{\frac{\pi}{2}}+1},
$$

hence $M_{\tau}$ has an exponential distribution with parameter $\sqrt{\frac{2}{\pi}}$. In Fig. 1, we compare the estimated density based on 100,000 samples generated from the algorithm, to the theoretical density of $M_{\tau}$, and the results are very close. For $\tau$, we compare the estimated density from the simulations to the density computed using the recursive formula derived in Dassios and Lim (2015).

We also check that the first and second moments of the simulated samples correspond to that of the $\operatorname{Exp}\left(\sqrt{\frac{2}{\pi}}\right)$ distribution with an error of $\pm 0.05 \%$ for the mean and variance of $M_{\tau}$. Furthermore, setting $\gamma=0$, we can also calculate the first moment of the distribution of $E(\tau)=2$, which corresponds to the numerical simulated result with an error of approximately $\pm 0.05 \%$.

Figure 2 presents the joint density of $\tau$ and $M_{\tau}$ estimated from the simulated samples.

\section{Application: Pricing Drawdown Options}

In this section, we propose a Monte Carlo method for pricing drawdown options using the simulation algorithm. We assume the Black-Scholes framework where the underlying asset $S_{t}$ is a Geometric Brownian motion under the risk-neutral measure $\mathcal{Q}$ :

$$
d S_{t}=S_{t}\left(r d t+\sigma d W_{t}\right), S_{0}=x,
$$
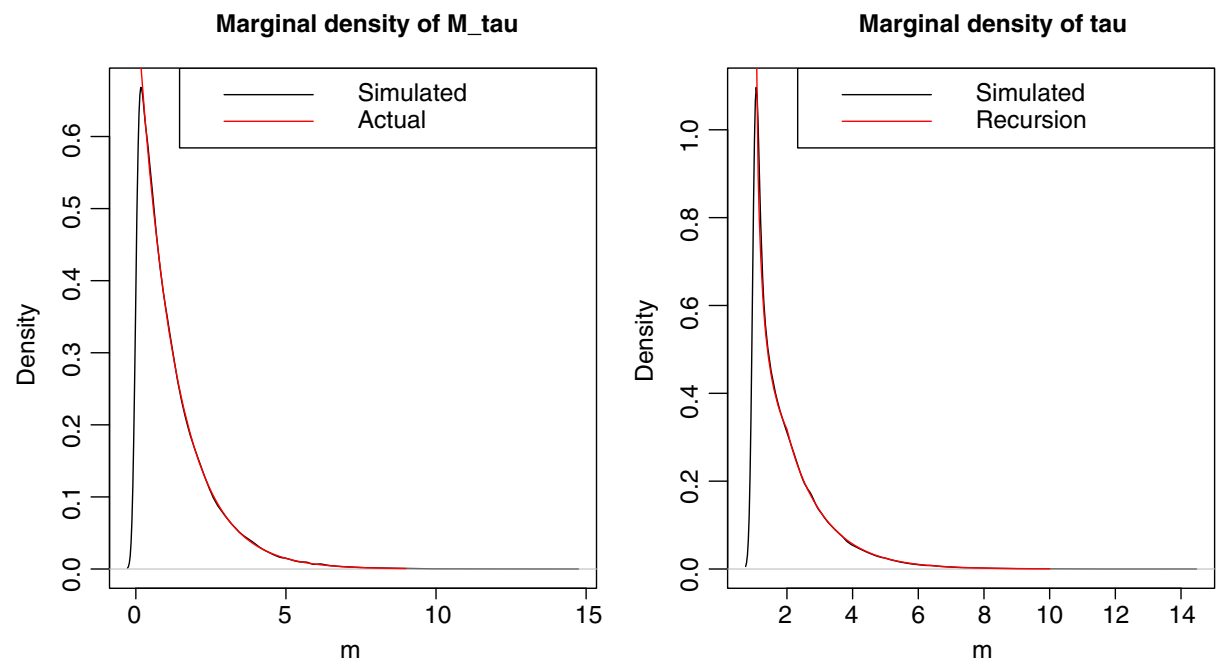

Fig. 1 Comparison of theoretical and simulated densities of $M_{\tau}$ and $\tau$ 


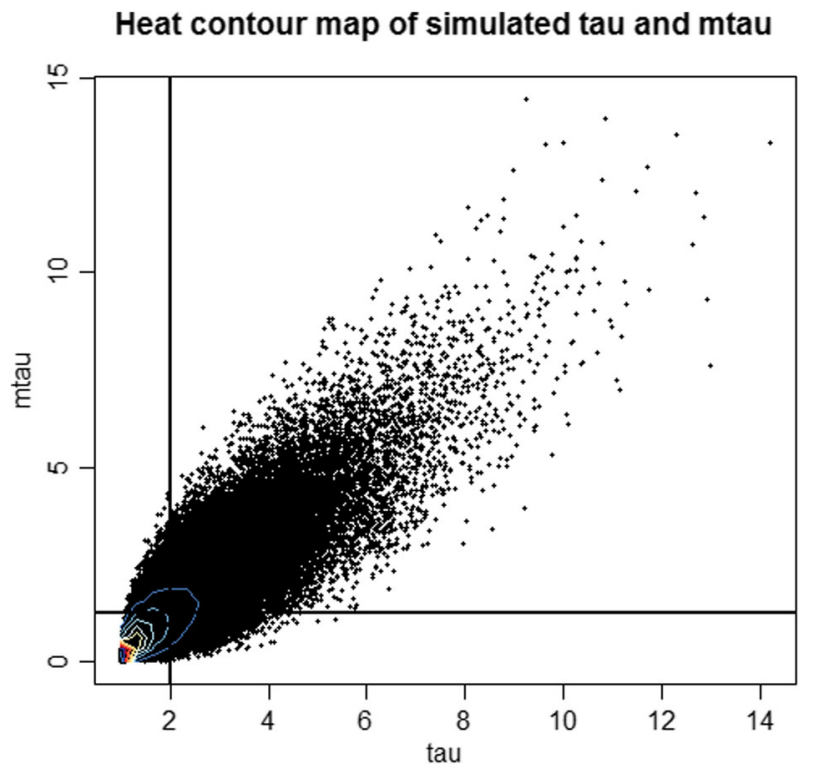

Fig. 2 Heat contour map of $\tau$ and $M_{\tau}$

so that

$$
S_{t}=S_{0} e^{\sigma W_{t}^{\mu}}
$$

where

$$
\begin{aligned}
W_{t}^{\mu} & =\mu t+W_{t}, \\
\mu & =\frac{1}{\sigma}\left(r-\frac{\sigma^{2}}{2}\right) .
\end{aligned}
$$

We also define

$$
\begin{aligned}
M_{t}^{\mu} & =\max _{0 \leq s \leq t} W_{s}^{\mu}, \\
\bar{S}_{t} & =\max _{0 \leq s \leq t} S_{s}=S_{0} e^{\sigma M_{t}^{\mu}}, \\
U_{t}^{S} & =t-\sup \left\{0 \leq s \leq t \mid \bar{S}_{t}=S_{s}\right\}=U_{t}, \\
\tau^{S} & =\inf \left\{t \geq 0 \mid U_{t}^{S}=1\right\}=\tau^{\mu} .
\end{aligned}
$$

As before we have taken the drawdown length $D=1$ without loss of generality. In order to price the option, we also need the result on the density of the Brownian motion with drift,

$$
P\left(\tau^{\mu} \in d t, M_{\tau}^{\mu} \in d m\right)=e^{\mu m} e^{-\frac{1}{2} \mu^{2} t} f(t, m)\left(1-\sqrt{2 \pi} \mu e^{\frac{m u^{2}}{2}} \mathcal{N}(-\mu)\right),
$$

where $f(t, m)$ is the joint density of $\tau$ and $M_{\tau}$ for a standard Brownian motion. Hence, any integral involving the density of $\left(\tau^{\mu}, M_{\tau}^{\mu}\right)$ can be written as an integral involving the density $f(t, m)$ and hence can be estimated using Monte Carlo simulations. 


\subsection{Single Drawdown Option}

We consider an option which pays off an amount proportional to the running maximum of the underlying asset when the drawdown duration first reaches length $D$. The discounted payoff of this option is $e^{-r \tau^{S}} \bar{S}_{\tau} s \mathbf{1}_{\left\{\tau^{S} \leq T\right\}}$ at time $\tau^{S}$. The price of the option at the start of the option lifetime, $P_{0}\left(S_{0}, T\right)$ is,

$$
\begin{aligned}
P_{0}\left(S_{0}, T\right) & =E_{\mathcal{Q}}\left(e^{-r \tau^{\mu}} S_{0} e^{\sigma M_{\tau}^{\mu}} \mathbf{1}_{\left\{\tau^{\mu} \leq T\right\}}\right) \\
& =\int_{0}^{T} \int_{0}^{\infty} e^{-r t} S_{0} e^{\sigma m} e^{\mu m} e^{-\frac{1}{2} \mu^{2} t} f(t, m)\left(1-\sqrt{2 \pi} \mu e^{\frac{\mu^{2}}{2}} \mathcal{N}(-\mu)\right) d t d m .
\end{aligned}
$$

This can be computed using Monte Carlo simulation, sampling from the distribution $f(t, m)$ using Algorithm 3.1.

Let $P_{t}\left(S_{t}, \bar{S}_{t}, U_{t}^{S}, T\right)$ denote the price at time $t$ of a drawdown option. It depends on the current stock price $S_{t}$, the current maximum $\bar{S}_{t}$, the current drawdown duration $U_{t}^{S}$, and the maturity time $T$. To price this option at time $t$, given that the drawdown has not achieved a duration of length 1 before time $t\left(\tau^{S}>t\right)$, and that the remaining lifetime of the option is sufficient for it to have a chance of getting a payoff $\left(1-U_{t}^{S} \leq T-t\right)$, we consider two cases, when the drawdown duration reaches length 1 in the current drawdown period, and when the underlying goes back to its maximum before the drawdown stopping time is hit. Let $T_{x}^{\mu}$ denote the hitting time of level $x$ of a Brownian motion with drift $\mu$. Then the price of the option is

$$
\begin{aligned}
P_{t}( & \left.S_{t}, \bar{S}_{t}, U_{t}^{S}, T\right) \\
= & E_{\mathcal{Q}}\left(e^{-r\left(\tau^{S}-t\right)} \bar{S}_{\tau} \mathbf{1}_{\left\{\tau^{S} \leq T\right\}} \mid S_{t}, \bar{S}_{t}, U_{t}^{S}, \tau^{S}>t\right) \\
= & E_{\mathcal{Q}}\left(\mathbf{1}_{\left\{T_{y}^{\mu}>1-U_{t}^{S}\right\}} e^{-r\left(1-U_{t}^{S}\right)} \bar{S}_{t} \mid S_{t}, \bar{S}_{t}, U_{t}^{S}, \tau^{S}>t\right) \\
& +E_{\mathcal{Q}}\left(\mathbf{1}_{\left\{T_{y}^{\mu} \leq 1-U_{t}^{S}\right\}} e^{-r\left(T_{y}^{\mu}+\tau^{\mu}\right)} \bar{S}_{t} e^{\sigma M_{\tau}^{\mu}} \mathbf{1}_{\left\{T_{y}^{\mu}+\tau^{\mu} \leq T-t\right\}} \mid S_{t}, \bar{S}_{t}, U_{t}^{S}, \tau^{S}>t\right) \\
= & \mathcal{Q}\left(T_{y}^{\mu}>1-U_{t}^{S}\right) e^{-r\left(1-U_{t}^{S}\right)} \bar{S}_{t} \\
& +E_{\mathcal{Q}}\left(\mathbf{1}_{\left\{T_{y}^{\mu} \leq 1-U_{t}^{S}\right\}} \bar{S}_{t} e^{-r T_{y}^{\mu}} E_{\mathcal{Q}}\left(e^{-r \tau^{\mu}} e^{\sigma M_{\tau}^{\mu}} \mathbf{1}_{\left\{\tau^{\mu} \leq T-t-T_{y}^{\mu}\right\}} \mid \mathcal{F}_{t+T_{y}^{\mu}}\right)\right)
\end{aligned}
$$

where $y=\frac{1}{\sigma} \log \left(\frac{\bar{S}_{t}}{S_{t}}\right)$ to simplify notation. The time it takes for $S$ to return to the running maximum $\bar{S}_{t}$ is $T_{y}^{\mu}$, and by the strong Markov property of Brownian motion, since it resets itself at $t+T_{y}^{\mu}$ at level $\bar{S}_{t},\left(\tau^{\mu}, M_{\tau}^{\mu}\right)$ is the drawdown stopping time and maximum of a Brownian motion starting at 0 again and can be generated independently from $T_{y}^{\mu}$. The hitting time $T_{y}^{\mu}$ has an inverse Gaussian distribution, $T_{y}^{\mu} \sim I G\left(\frac{y}{\mu}, y^{2}\right)$. The second term (5.17) can thus be computed using Monte Carlo simulation, sampling from an inverse Gaussian distribution $T_{y}^{\mu}$ and independently, the pair $\left(\tau, M_{\tau}\right)$.

\subsection{Multiple Drawdown Option}

This simulation method is particularly effective in multivariate cases where multiple integrals need to be evaluated. To demonstrate this advantage, we introduce multiple drawdown 
options, a new type of drawdown option which provide insurance against prolonged drawdowns each time it occurs. These options pay off an amount proportional to the running maximum every time the drawdown duration reaches length 1 , until the option expires. Pricing using the recursive formulas in Dassios and Lim (2015) would become too cumbersome when pricing multiple drawdown options, as an additional integral would need to be evaluated for each drawdown time. This simulation algorithm, however, eliminates the need to evaluate multiple integrals.

Let $\tau_{1}=\tau^{S}$ and define the series of stopping times $\tau_{i}=\inf \left\{t>\tau_{i-1} \mid U_{t}^{S}=1\right\}$. We first look an option which pays off an amount proportional to the running maximum at the second time the drawdown duration reaches length 1 . This option has discounted payoff $e^{-r \tau_{2}} \bar{S}_{\tau_{2}} \mathbf{1}_{\left\{\tau_{2} \leq T\right\}}$ at time $\tau_{2}$. Hence, the price of the option is

$$
\begin{aligned}
P_{0}^{2}\left(S_{0}, T\right) \\
=E_{\mathcal{Q}}\left(e^{-r \tau_{2}} \bar{S}_{\tau_{2}} \mathbf{1}_{\left\{\tau_{2} \leq T\right\}}\right) \\
=E_{\mathcal{Q}}\left(E_{\mathcal{Q}}\left(e^{-r \tau_{2}} \bar{S}_{\tau_{2}} \mathbf{1}_{\left\{\tau_{2} \leq T\right\}} \mid \mathcal{F}_{\tau_{1}}, \tau_{1} \leq T-1\right)\right) \\
=E_{\mathcal{Q}}\left(E_{\mathcal{Q}}\left(e^{-r\left(\tau_{1}+T_{y}^{\mu}+\tau^{\mu}\right)} \bar{S}_{\tau_{1}} e^{\sigma M_{\tau} \mu} \mathbf{1}_{\left\{\tau_{1}+T_{y}^{\mu}+\tau^{\mu} \leq T\right\}} \mathbf{1}_{\left\{\tau_{1}+T_{y}^{\mu} \leq T-1\right\}} \mid \mathcal{F}_{\tau_{1}}, \tau_{1} \leq T-1\right)\right) \\
=E_{\mathcal{Q}}\left(e^{-r \tau_{1}} \bar{S}_{\tau_{1}} E_{\mathcal{Q}}\left(e^{-r\left(T_{y}^{\mu}+\tau^{\mu}\right)} e^{\sigma M_{\tau}^{\mu}} \mathbf{1}_{\left\{\tau_{1}+T_{y}^{\mu}+\tau^{\mu} \leq T\right\}} \mathbf{1}_{\left\{\tau_{1}+T_{y}^{\mu} \leq T-1\right\}} \mid \mathcal{F}_{\tau_{1}}, \tau_{1} \leq T-1\right)\right) \\
=E_{\mathcal{Q}}\left(e ^ { - r \tau _ { 1 } } \overline { S } _ { \tau _ { 1 } } \mathbf { 1 } _ { \{ \tau _ { 1 } \leq T - 1 \} } E _ { \mathcal { Q } } \left(e^{-r T_{y}^{\mu}} \mathbf{1}_{\left\{T_{y}^{\mu} \leq T-1-\tau_{1}\right\}} E_{\mathcal{Q}}\right.\right. \\
\left.\left.\quad \times\left(e^{-r \tau^{\mu}} e^{\sigma M_{\tau}^{\mu}} \mathbf{1}_{\left\{\tau^{\mu} \leq T-\tau_{1}-T_{y}^{\mu}\right\}} \mid \mathcal{F}_{\tau_{1}+T_{y}^{\mu}}\right) \mid \mathcal{F}_{\tau_{1}}\right)\right),
\end{aligned}
$$

\begin{tabular}{|c|c|c|c|c|c|c|}
\hline \multirow[b]{2}{*}{$\mathrm{T}$} & \multicolumn{2}{|c|}{$D=1$ year } & \multicolumn{2}{|c|}{$D=1 / 2$ year } & \multicolumn{2}{|c|}{$D=1 / 4$ year } \\
\hline & $S_{0}=50$ & $S=100$ & $S_{0}=50$ & $S_{0}=100$ & $S_{0}=50$ & $S_{0}=100$ \\
\hline 2 & 29.89 & 61.51 & 62.25 & 123.48 & 112.17 & 226.95 \\
\hline 3 & 44.96 & 95.91 & 91.28 & 183.27 & 156.60 & 314.46 \\
\hline 4 & 63.38 & 128.13 & 120.74 & 242.23 & 195.81 & 387.49 \\
\hline 5 & 80.41 & 160.63 & 149.40 & 298.81 & 233.81 & 465.64 \\
\hline 6 & 96.48 & 194.72 & 176.79 & 351.90 & 266.44 & 531.92 \\
\hline 7 & 116.60 & 230.65 & 200.03 & 401.17 & 303.73 & 606.97 \\
\hline 8 & 132.41 & 265.43 & 222.85 & 449.22 & 341.71 & 677.72 \\
\hline 9 & 150.36 & 299.94 & 248.98 & 501.95 & 372.91 & 753.51 \\
\hline 10 & 167.77 & 337.55 & 274.23 & 544.66 & 421.08 & 836.10 \\
\hline
\end{tabular}

with $y=M_{\tau_{1}}-W_{\tau_{1}}$ here. The pair $\left(\tau^{\mu}, M_{\tau}^{\mu}\right)$ are the stopping time and maximum after the Brownian motion resets at level $M_{\tau_{1}}$ at time $\tau_{1}+T_{y}^{\mu}$, and following from the strong Markov property of Brownian motion, they can be generated independently from $\tau_{1}$ and $T_{y}^{\mu}$. We generate first the triplet $\left(\tau_{1}, M_{\tau_{1}}, W_{\tau_{1}}\right)$, followed by $T_{y}^{\mu}$ and then the pair $\left(\tau^{\mu}, M_{\tau}^{\mu}\right)$. The price of the option can then be computed by aggregating a function of these variables.

Table 1 Price of Multiple Drawdown Options, $P_{M D}\left(S_{0}, T\right)$ 
Similarly, by induction, we can see that the price of the $k^{\text {th }}$ drawdown option can be computed from the $k-1^{\text {th }}$ drawdown stopping time using the formula

$$
\begin{aligned}
P_{0}^{k}\left(S_{0}, T\right) & =E_{\mathcal{Q}}\left(e^{-r \tau_{k}} \bar{S}_{\tau_{k}} \mathbf{1}_{\left\{\tau_{k} \leq T\right\}}\right) \\
= & E_{\mathcal{Q}}\left(e ^ { - r \tau _ { k - 1 } } \overline { S } _ { \tau _ { k - 1 } } \mathbf { 1 } _ { \{ \tau _ { k - 1 } \leq T - 1 \} } E _ { \mathcal { Q } } \left(e^{-r T_{y}^{\mu}} \mathbf{1}_{\left\{T_{y}^{\mu} \leq T-1-\tau_{k-1}\right\}}\right.\right. \\
& \left.\left.E_{\mathcal{Q}}\left(e^{-r \tau^{\mu}} e^{\sigma M_{\tau}^{\mu}} \mathbf{1}_{\left\{\tau^{\mu} \leq T-\tau_{k-1}-T_{y}^{\mu}\right\}} \mid \mathcal{F}_{\tau_{k-1}+T_{y}^{\mu}}\right) \mid \mathcal{F}_{\tau_{k-1}}\right)\right),
\end{aligned}
$$

with $y=M_{\tau_{k-1}}-W_{\tau_{k-1}}$. For multiple drawdown options, there are multiple payoffs. The price of the option is

$$
P_{0}^{M D}\left(S_{0}, T\right)=E_{\mathcal{Q}}\left(\sum_{i=1}^{\infty} e^{-r \tau_{i}} \bar{S}_{\tau_{i}} \mathbf{1}_{\left\{\tau_{i} \leq T\right\}}\right),
$$

and each term in the summation can be computed recursively, generating a sample from $\left(\tau_{i}, M_{\tau_{i}}, W_{\tau_{i}}\right)$ and $T_{y}^{\mu}$ each time. The price can then be computed by aggregating across all the samples. Table 1 presents the prices of the multiple drawdown options with drawdown duration 1 and a range of maturities.

Open Access This article is distributed under the terms of the Creative Commons Attribution 4.0 International License (http://creativecommons.org/licenses/by/4.0/), which permits unrestricted use, distribution, and reproduction in any medium, provided you give appropriate credit to the original author(s) and the source, provide a link to the Creative Commons license, and indicate if changes were made.

\section{References}

Anderluh JHM (2008) Pricing Parisians and barriers by hitting time simulation. Eur J Fin 14(2):137-156

Bernard C, Boyle P (2011) Monte Carlo methods for pricing discrete Parisian options. Eur J Fin 17(3):169_ 196

Carr P, Zhang H, Hadjiliadis O (2011) Maximum drawdown insurance. Int J Theor Appl Fin 14(8):11951230

Chesney M, Jeanblanc-Picqué M, Yor M (1997) rownian excursions and Parisian barrier options. Adv Appl Probab 29(1):165-184

Dassios A, Wu S (2010) Perturbed Brownian motion and its application to Parisian option pricing. Fin Stoch 14:473-494

Dassios A, Lim JW (2013) Parisian option pricing: a recursive solution for the density of the parisian stopping time. SIAM J Fin Math 4(1):599-615

Dassios A, Lim JW (2015) An analytical solution for the two-sided Parisian stopping time, its asymptotics and the pricing of Parisian options. Math Fin 00(00):1-17

Dassios A, Lim JW (2016). An extension to the Azéma martingale and drawdown options

Douady R, Shiryaev AN, Yor M (2000) On probability characteristics of "downfalls" in a standard Brownian motion. Theory Probab Appl 44(1):29-38

Landriault D, Li B, Zhang H (2015) On the frequency of drawdowns for Brownian motion processes. J Appl Probab 52(1):191-208

Landriault D, Li B, Zhang H (2017) On magnitude, asymptotics and duration of drawdowns for Levy models. Bernoulli 23(1):432-458

Taylor H (1975) A stopped Brownian motion formula. Ann Probab 3:234-246

Zhang H, Hadjiliadis O (2012) Drawdowns and speed of market crash. Methodol Comput Appl Probab 14(3):739-752 